\title{
Uniqueness and You: How the Disorder of Atomic Structure Enables Your World
}

\author{
Katharine Page ${ }^{1}$, Daniel Olds ${ }^{1,2}$, and Thomas Proffen ${ }^{2}$ \\ ${ }^{1}$ Chemical and Engineering Materials Division, ${ }^{2}$ Neutron Data Analysis and Visualization \\ Division, Oak Ridge National Laboratory, Oak Ridge, TN 37831
}

Crystallographers are trained to examine the world of materials structures for exploitable traits, to help scientists from diverse disciplines understand the inner-workings of property mechanisms, and ultimately to optimize and control them through atomic structure modification. For example, defects and chemical ordering at the surface of metal oxide nanoparticles can be optimized to more efficiently recycle greenhouse gas. Defect structure stories are at the heart of data storage, iron-based superconductor, lithium-ion battery, and solid-state lighting technologies, to name a few. In each of these cases a series of structural motifs have been identified, modified, and optimized to reach the levels of performance, resource-savings, environmental stewardship, and materials safety we enjoy today. In this contribution we highlight a crystallography outreach activity for middle school grades, aimed at communicating the centrality of underlying defects and disorder to materials functionality. Our one hour classroom program consists of simple structure-property vignettes demonstrating cause-andeffect relationships, hands-on exploration through structure model building, and an interactive computer program in which students explore simulated and real diffusing scattering data through a simplified refinement and visualization interface. Our project communicates parallels between the unique traits that distinguish individuals, and the atomic level defects and disorder that enable exciting new technologies, both at great benefit to society. 